\title{
COVID-19 and Warburg phenomenon: pulmonary vasopleagia or vasoconstriction?
}

Amit Jain ${ }^{1}$

${ }^{1}$ Affiliation not available

June 11, 2020

Author Information: Amit Jain M.D., D. John Doyle, M.D., Ph.D.

Amit Jain M.D., Anesthesiology Institute, Cleveland Clinic Abu Dhabi, United Arab Emirates

D. John Doyle, M.D., Ph.D., Anesthesiology Institute, Cleveland Clinic Abu Dhabi, United Arab Emirates

\section{Corresponding Author:}

Amit Jain

Anesthesiology Institute, Cleveland Clinic Abu Dhabi, Al Maryah Island, Abu Dhabi

United Arab Emirates

M: +971544103790

Email:amitvasujain@gmail.com

Word and Element Counts:

Word Count: 500

Number of references: 5

Number of figures: 1

Funding Statement: Support was provided solely from institutional and/or departmental sources

Conflicts of Interest: The authors declare no competing interests

To the Editor:

The article by Archer et al. ${ }^{1}$ proposes a novel COVID-19 pathobiology: mitochondrial oxygen-sensing failureinduced loss of hypoxic pulmonary vasoconstriction (HPV) and impaired carotid body function, leading to "happy hypoxemia". We write to propose an alternate model (Figure 1.) favoring pulmonary vasoconstriction $(\mathrm{PV})$, with apoptotic-resistant, proliferative angiogenesis that may shift to "reperfusion-hyperoxia" model of cell-injury and inflammation in more severe stage of COVID-19 pneumonia.

1. Warburg phenomenon and pseudohypoxic state: SARS-CoV-2 causes downregulation of ACE2 and overactivity of ACE-Ang II-AT1R. AII induces epigenetic reduction, via Akt/mTOR/FOXO signaling, in mitochondrial SOD2 expression, decreases $\mathrm{H} 2 \mathrm{O} 2$ production and creates a pseudohypoxic state. AII also activates Erk 1/2 resulting in increased expression of DNA methyltransferase (DNMT). DNMT-mediated changes in redox signaling causes normoxic activation of HIF-1 $\alpha$ and increases pyruvate dehydrogenase kinase (PDK) expression. PDK phosphorylates and inhibits pyruvate 
dehydrogenase, increasing uncoupled aerobic glycolysis (Warburg phenomenon). ${ }^{2}$ Warburg metabolism changes one or more mitochondrial metabolic pathways, altering aerobic glycolysis or fatty-acid oxidation and/or induction of glutaminolysis. ${ }^{2}$ SARS-CoV-2 facilitates Akt1-dependent degradation of autophagy and a switch from oxidative-phosphorylation to lactic-acid formation. ${ }^{3}$ This results in upregulation of glutathione metabolism, limiting mitochondrial-derived redox signaling, ${ }^{3}$ creating a pseudohypoxic state and HIF-1 $\alpha$ upregulation.

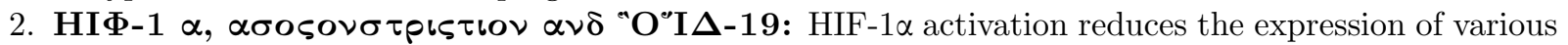
ion channels, including Kv1.5, leading to depolarized, calcium-overloaded pulmonary artery smooth muscle (PASM) producing PV. ${ }^{2}$ High levels of HIF-1 $\alpha$ gene expression along with other angiogenesisassociated genes including VEGFs in lungs from COVID-19 patients supports a hyperproliferative vasoactive model. ${ }^{4}$

3. Mitophagy: Altered mitochondria-derived ROS production (high $\mathrm{O}_{2}^{-}$, low $\mathrm{H}_{2} \mathrm{O}_{2}$ ), increased mitochondrial membrane potentials $\left(\Delta \psi_{\mathrm{m}}\right)$, and mitochondrial morphology (increased mitotic fission) favors a hyperproliferative, apoptosis-resistant phenotype in PASM, endothelial cells, and fibroblasts, contributing to obstructive vasculopathy ${ }^{2}$.

4. Ang II and NOXs: Ang II-AT1R-NOX-ROS derived cytoplasmic ROS triggers the opening of mitochondrial $\mathrm{K}_{\mathrm{ATP}}$ channels and mitochondrial ROS production in positive feedback loop, thus developing the "reperfusion-hyperoxia" model of cell-injury resulting in an abrupt collapse of $\Delta \psi_{\mathrm{m}}$, and mitochondrial death. Ang II has also been implicated in decreasing the activity of scavenging enzymes. In endothelial cells, increased $\mathrm{O}^{-}$inactivate the vasodilator $\mathrm{NO}$ by uncoupling of eNOS, leading to endothelial-dysfunction and PV.

We speculate that the hypoxemia in early COVID-19 pneumonia is from heterogenous PV and recruitment of pulmonary capillaries experiencing relatively less vasoconstriction, causing regional over-perfusion, reduced diffusion-capacity, and increased shunt. This explains the benefits of prone ventilation in COVID-19 patients compared to typical ARDS.

In both COVID-19 and high-altitude pulmonary edema (HAPE), patients typically appear better than expected given the hypoxemia severity. ${ }^{5}$ However, we speculate that COVID-19 and HAPE behave differently due to differences in PV patterns, with the severity of PAH diminished in COVID-19. The initial presentation of respiratory alkalosis in COVID-19 suggests intact carotid body sensing with functioning ventilatory mechanics early in the disease producing "silent hypoxia".

Thus, pathological PV (with dysregulated HPV) instead of pulmonary vasoplegia should be a plausible mechanism for severe hypoxia in COVID-19 pneumonia. Moreover, orthodeoxia and platypnea, the usual signs of pulmonary vasoplegia are not seen in COVID-19 patients.

\section{References:}

1. Archer SL, Sharp WW, Weir EK. Differentiating COVID-19 Pneumonia from Acute Respiratory Distress Syndrome (ARDS) and High Altitude Pulmonary Edema (HAPE): Therapeutic Implications [published online ahead of print, 2020 May 5]. Circulation. 2020;10.1161/CIRCULATIONAHA.120.047915.doi:10.1161/CIRCULATIONAHA.120.047915

2. Dasgupta A, Wu D, Tian L, et al. Mitochondria in the Pulmonary Vasculature in Health and Disease: Oxygen-Sensing, Metabolism, and Dynamics. Compr Physiol. 2020;10(2):713-765. Published 2020 Mar 12. doi:10.1002/cphy.c190027

3. Gassen NC, Papies J, Bajaj T, Dethloff F, Emanuel J, Weckmann K, et al. Analysis of SARS-CoV-2controlled autophagy reveals spermidine, MK-2206, and niclosamide as putative antiviral therapeutics. published online April 15, 2020. bioRxiv 2020. doi: https://doi.org/10.1101/2020.04.15.997254

4. Ackermann M, Verleden SE, Kuehnel M, et al. Pulmonary Vascular Endothelialitis, Thrombosis, and Angiogenesis in Covid-19 [published online ahead of print, 2020 May 21]. N Engl J Med. 2020;10.1056/NEJMoa2015432. doi:10.1056/NEJMoa2015432

5. Hackett PH, Roach RC. High-altitude illness. N Engl J Med. 2001;345(2):107-114. doi:10.1056/NEJM200107123450206 


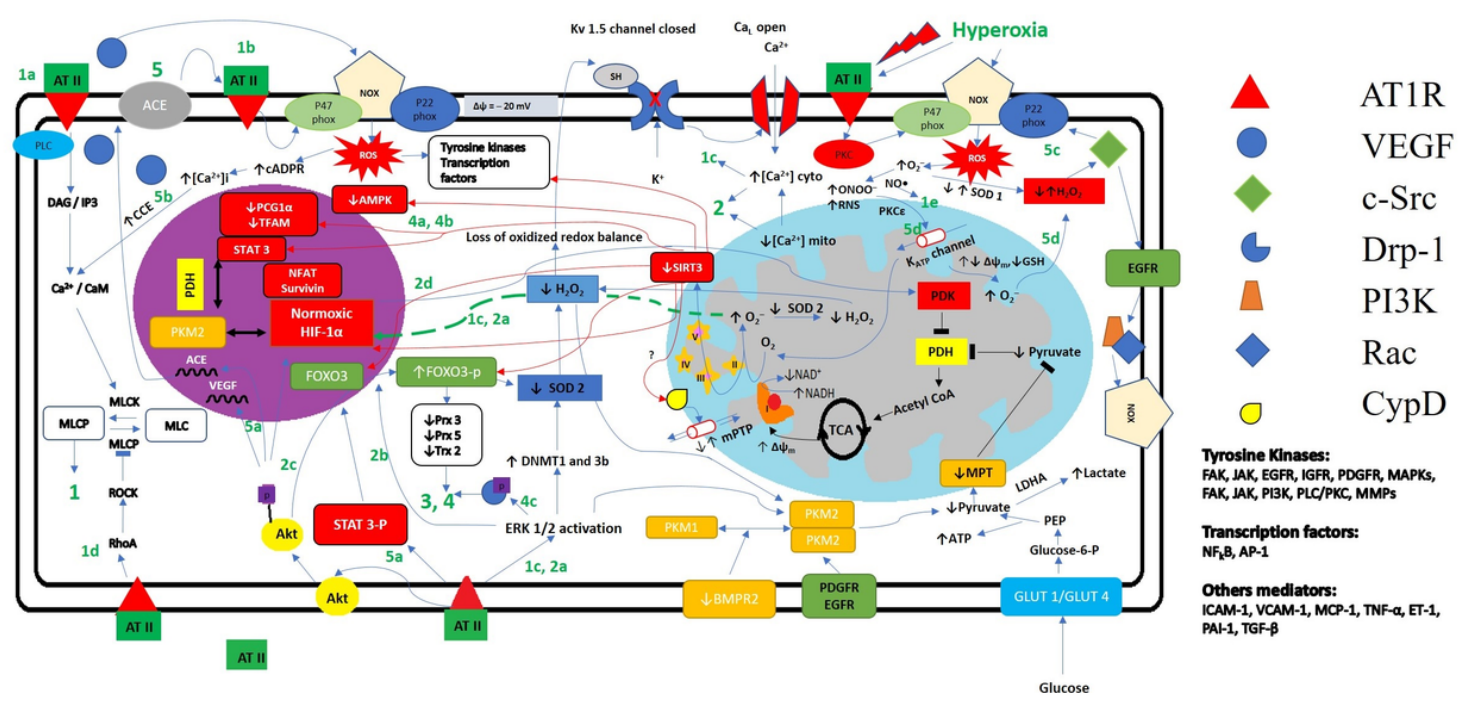

Figure 1. Schematic diagram for proposed pathophysiology of COVID-19 pneumonia: an uncontrolled Ang II overactivity-mediated pulmonary vasoconstriction, Warburg phenomenon and reperfusion-hyperoxia model

1. Ang II mediated pathways involved in pulmonary vasoconstriction: increased cytosolic calcium via direct:(1a) Ang II/AT1R/PLC/Ca2+-Calmodulin pathway) or indirect pathways: (1b) AT1R/NOX/ROS/cADPR/CCE/Ca2+ pathway;(1c) Ang II/AT1R/Erk1/2/DNMT/SOD2 pathway (pseudohypoxia) results in activation of myosin light chains. This along with Rho-mediated inhibition of myosin light chain phosphatases result in actin-myosin coupling and vasoconstriction (1d) Ang II/AT1R/Rho/ROCK pathway (1e) Increased superoxide-mediated uncoupling of NO results in reduced bioavailability of NO and inhibits vasodilation and produce pro-thrombotic activity.

2. Warburg phenomenon and pseudohypoxia state: (2a) Ang II/AT1R/Erk1/2/DNMT/SOD2/HIF 1 $\alpha /$ PDK pathway induces hypermethylation of a CpG island in the SOD2 promoter results reduced SOD 2 activity, decreased mitochondrial $\mathrm{H}_{2} \mathrm{O}_{2}$ production with increase in mitochondrial superoxide levels resulting in normoxaic stabilization of HIF $1 \alpha$ and uncoupled glycolysis. Reduced $\mathrm{H}_{2} \mathrm{O}_{2}$ is coupled with less oxidized redox state, closure of Kv channels with increased opening of type- $\mathrm{L} \mathrm{Ca}^{2+}$ channels and raised cytosolic $\mathrm{Ca}^{2+}$ influx with decreased mito $\mathrm{Ca}^{2+}$. Other pathways that results in stabilization of HIF1 $\alpha$ : (2b) Ang-II/Erk2/Dpr-1 pathway (2c)PI3K/Akt/FOXO3/SOD2 pathway and (2d) NOX/ROS/SIRT 3/FOXO 3/SOD 2 pathway

3. Mitochondrial biogenesis impairment: Reduced SITR3 activity results in reduced acetylation of FOXO3 and increased phosphorylated form of FOXO3. This reduced nuclear translocation of FOXO3 with reduced transcription of antioxidant enzymes (SOD2, Prx 3, Prx 5, Trx 2). Unopposed superoxide activity also results in impaired mitochondrial biogenesis

4. Increased mitochondrial fission/reduced mitochondrial fusion:reduced SIRT 3/PGC$1 \alpha /$ TFAM and (4a) increased (4b)STAT 3 pathways activates Dynamin-related protein (Drp-1) activating kinases including Erk 2 and results in Drp-1 mediated increased mitochondrial fission, impaired apoptosis and increased proliferative angiogenesis. (4c) Ang-II/Erk2/Dpr-1 pathway

5. Positive feed-forward or feed-back pathways resulting in vicious cycle: (5a) AT1R/HIF$1 \alpha / \mathrm{ACE} / \mathrm{Ang} \mathrm{II} / \mathrm{Ca}^{2+}$ loop via PI3K/Akt pathway and STAT3 pathway fostering VEGF synthesis(5b) $\mathrm{Ca} 2+$ mediated $\mathrm{Ca}^{2+}$ entry further increasing cytosolic $\mathrm{Ca}^{2+}$ (5c) A II/NOX/ROS/c-Src/NOX pathway and (5d) Ang II/NOX/ROS/RNS/mtK opening of mPTP channels and abrupt collapse of $\Delta \psi \mathrm{m}$. RNS are associated with increased cytotoxic 
effects and platelet activation (not described in the Figure) 